Copyright@2007 IEEE. Reprinted from:

F. Belloni, A. Richter, and V. Koivunen, "DoA Estimation via Manifold Separation for Arbitrary Array Structures," IEEE Transactions on Signal Processing, vol. 55 no. 10, pp. 4800-4810, October 2007.

This material is posted here with permission of the IEEE. Such permission of the IEEE does not in any way imply IEEE endorsement of any of Helsinki University of Technology's products or services. Internal or personal use of this material is permitted. However, permission to reprint/republish this material for advertising or promotional purposes or for creating new collective works for resale or redistribution must be obtained from the IEEE by writing to pubs-permissions@ieee.org.

By choosing to view this document, you agree to all provisions of the copyright laws protecting it. 


\title{
DoA Estimation Via Manifold Separation for Arbitrary Array Structures
}

\author{
Fabio Belloni, Student Member, IEEE, Andreas Richter, Member, IEEE, and Visa Koivunen, Senior Member, IEEE
}

\begin{abstract}
In this paper, we consider the manifold separation technique (MST), which stems from the wavefield modeling formalism developed for array processing. MST is a method for modeling the steering vector of antenna arrays of practical interest with arbitrary 2-D or 3-D geometry. It is the product of a sampling matrix (dependent on the antenna array only) and a Vandermonde structured coefficients vector depending on the wavefield only. This allows fast direction-of-arrival (DoA) algorithms designed for linear arrays to be used on arrays with arbitrary configuration. In real-world applications, the calibration measurements used to determine the sampling matrix are corrupted by noise. This impairs the performance of MST-based algorithms. In particular, we study the effect of noisy calibration measurements on subspace-based DoA algorithms using MST. Expressions describing the error in the DoA estimates due to calibration noise and truncation are derived. This allows predicting the performance of MST-based algorithms in real-world applications. The analysis is verified by simulations. We established a link between the optimal number of selected modes and the statistics of calibration noise. We analyze the modeling error when MST is used for 1-D (azimuth) DoA estimation.
\end{abstract}

Index Terms-Calibration measurement noise, direction-of-arrival (DoA) estimation, effective aperture distribution function (EADF), error analysis, manifold separation technique.

\section{INTRODUCTION}

$\mathbf{I}$ $\mathrm{N}$ array signal processing, it is often convenient to work with arrays having a steering vector matrix with a Vandermonde structure. For example, this allows using fast direction-of-arrival (DoA) estimators designed for uniform linear arrays (ULA) such as root-MUSIC (MUltiple SIgnal Classification), root-WSF (Weighted Subspace Fitting) [2], [6] [3], [5], and RARE (RAnk Reduction Estimator) [19]. Similarly, for uniform rectangular arrays (URAs), there exist computational efficient DoA estimation algorithms [23].

Techniques such as array interpolation [2]-[5] and beamspace transform [6], [7] have been developed in order to map the steering vectors of any planar array onto steering vectors of a ULA-type array called the virtual array. These preprocessing techniques often introduce mapping errors [4] that cause bias [3], [7] and excess variance [8] in the DoA estimates. Hence, the estimates are not statistically optimal.

Manuscript received August 15, 2006; revised January 29, 2007. The associate editor coordinating the review of this manuscript and approving it for publication was Dr. Petr Tichavsky. This work was supported by grants from the Academy of Finland (TULE program), Tekniikan edistämissäätiö (TES), Nokia and Wihuri Foundations.

The authors are with the SMARAD Center of Excellence, Signal Processing Laboratory, Department of Electrical and Communications Engineering, Helsinki University of Technology (TKK), FIN-02015 HUT, Finland (e-mail: fbelloni@wooster.hut.fi; arichter@wooster.hut.fi; visa@wooster.hut.fi; website: http://wooster.hut.fi).

Digital Object Identifier 10.1109/TSP.2007.896115
Here, we consider an alternative approach to this class of problems. We exploit the wavefield modeling formalism for array processing [13]-[15] in order to describe the manifold separation technique (MST) [9]-[16]. Wavefield modeling is based on the idea that the signal-dependent part of the array output can be rewritten by an array sampling operator, operating on the impinging wavefield. Using this approach, the steering vector of an arbitrary array can be modeled as the product of a characteristic matrix describing the array itself (sampling matrix) and a vector with a Vandermonde structure containing the unknown angular parameter (coefficient vector).

MST allows polynomial rooting-based techniques such as root-MUSIC and RARE to be applied with arbitrary 2-D or 3-D array configurations of practical interest [9]-[11], [13], [16]. In contrast to interpolation and mapping techniques, MST does not require any division into angular sectors [2], and it can provide a significantly smaller fitting error over the whole $360^{\circ}$ coverage area [10]. Furthermore, MST allows processing the data recorded by the array directly in element space [9]. Hence, no transformation or interpolation of the data is required, and mapping errors [3], [4], [7] can be avoided.

We consider a practical approach (based on a discrete Fourier transform) for computing the sampling matrix from array calibration measurements. The calibration data contains information on array nonidealities, such as mutual coupling, antenna manufacturing errors, sensors orientation, and position.

Calibration data are always corrupted by measurement noise. In order to reduce the impact of this noise on the MST, we use a truncated version of the sampling matrix, known as effective aperture distribution function (EADF) of the array [9], [10], [17], [18], [21]. A criterion for selecting the dimension of the EADF is presented, and a link between the optimum number of selected modes and the calibration noise power is established.

The influence of antenna array model mismatch on DoA estimation error has been analyzed in several papers (see [3], [4], [7], [24], [28], and references therein). In this paper, we analyze the error due to calibration noise in the modeling of the array steering vector when MST is used. This error causes an error floor in the DoA estimates, which can dominate over the (random) error due to observation noise and finite sample effects [3], [24].

We derive a closed-form expression for the error in DoA estimates due to calibration noise, assuming the true array manifold is known. In a second step, we derive an approximate expression for the mean square error (MSE) assuming that the calibration noise is random. This is of particular interest in practice because it depends only on quantities that are known or computable in real-world applications. Based on the developed analysis, it is possible to predict the performance limit achieved by subspace-based algorithms using MST. For the error analysis, 
we have used the element-space (ES) root-MUSIC algorithm [9], [10]. The results are verified by extensive simulations.

Throughout the paper, we assume that the noncoherent narrowband sources are located at the same (known) elevation angle. Hence, we consider 1-D (azimuth) angular estimation. An extension considering also the estimation of the polarization of the sources can be found in [11].

This paper is organized as follows. First, the signal model is defined, and the key assumptions are stated. In Section III, we give a brief overview of the conventional wavefield modeling, and we introduce the MST. In Section IV, we extend the MST by introducing the concept of EADF. In Section V, the ES-rootMUSIC algorithm is presented. In Section VI, we describe the impact of noisy calibration data on the EADF. In Section VII, an analysis of the calibration noise impact on DoA estimates is given, and expressions for computing the error are derived. In Section VIII, we verify the proposed error analysis by means of simulations. Finally, Section IX concludes the paper.

\section{SignAL ModEL}

Unless explicitly stated, we assume to have $N$, possibly coupled, sensors forming a 2-D or 3-D antenna array with an arbitrary geometry. Each element has its individual directional characteristic. For convenience, we define the origin of the coordinate system to be at the centroid of the array. Note that in practice the centroid is defined by the calibration setup.

The co-elevation angle $\theta$ is measured down from the $z$ axis (assumed to be fixed at $\theta=90^{\circ}$ ), and $\phi$ is the azimuth angle (unknown parameter) measured counterclockwise from the $x$ axis in the $x-y$ plane.

There are $P(P<N)$ noncoherent narrowband signal sources on the $x-y$ plane, impinging an array from directions $\boldsymbol{\phi}=\left\{\phi_{1}, \phi_{2}, \ldots, \phi_{P}\right\}$, where $\phi$ is the azimuth angle. Furthermore, we assume that $K$ snapshots are observed by the array. Let us define the array steering matrix $\mathbf{B}=\left[\mathbf{b}\left(\phi_{1}\right), \mathbf{b}\left(\phi_{2}\right), \ldots, \mathbf{b}\left(\phi_{P}\right)\right] \in \mathbb{C}^{N \times P}$, using the steering vectors $\mathbf{b}\left(\phi_{p}\right)=\left[b_{0}\left(\phi_{p}\right), b_{1}\left(\phi_{p}\right), \ldots, b_{N-1}\left(\phi_{p}\right)\right]^{T} \in \mathbb{C}^{N \times 1}$. The $\mathbf{X} \in \mathbb{C}^{N \times K}$ array data matrix may then be written as

$$
\mathbf{X}=\mathbf{B S}+\mathbf{N}
$$

where $\mathbf{S} \in \mathbb{C}^{P \times K}$ is the signal matrix with $\operatorname{rank}\left(\mathbf{S S}^{H}\right)=P$, and $\mathbf{N} \in \mathbb{C}^{N \times K}$ contains the observation noise. The noise is modeled as a stationary, second-order ergodic, zero-mean, spatially and temporally white circular complex Gaussian process.

\section{WAVEFIELD MODELING AND MANIFOLD SEPARATION}

Here, we give a brief overview of wavefield modeling [13]-[15]. This forms the theoretical basis of our contribution. We will use it for justifying and explaining several aspects of the proposed array modeling technique based on the EADF.

We assume that the wavefield is generated by a far field source only (plane wave assumption) and that the sampling operator is linear, smooth and continuous. Note, the latter conditions are not restrictive because they are satisfied by real-world (physical) antenna arrays [13]. Furthermore, at this stage, we consider an array formed with omnidirectional sensors. This assumption does not restrict the generality of the discussion and the results can be extended to the case of directional sensors, as shown in [13].

Based on the model in [12], we write the $n$th element of the array manifold for arrays with arbitrary configuration having omnidirectional sensors as

$$
[\mathbf{b}(\phi)]_{n}=e^{-j \omega \tau_{n}(\phi)}=e^{j \kappa r_{n} \cos \left(\gamma_{n}-\phi\right)}
$$

where $\kappa=(\omega / c)$ is the wavenumber, $\omega=2 \pi f$ is the angular frequency, and $\tau_{n}(\phi)=-\left(r_{n} / c\right) \cos \left(\gamma_{n}-\phi\right)$ is the propagation delay associated with the $n$th sensor and a wavefront impinging from direction $\phi$. Here, $r_{n}$ is the distance from the centroid of the array and $\gamma_{n}$ is the angular position (counted counterclockwise from the $x$ axis) of the $n$th array element in polar coordinates.

By using the Jacobi-Anger expansion [20], we can mathematically express (2) as [12], [13], [16]

$$
\begin{aligned}
& e^{j \kappa r_{n} \cos \left(\gamma_{n}-\phi\right)} \\
& \quad=\sum_{m=-\infty}^{\infty} j^{m} J_{m}\left(\kappa r_{n}\right) e^{j m\left(\gamma_{n}-\phi\right)} \\
& \quad=\frac{1}{\sqrt{2 \pi}} \sum_{m=-\infty}^{\infty}\left[\mathbf{G}_{s}\left(r_{n}, \gamma_{n}\right)\right]_{n, m} e^{-j m \phi}
\end{aligned}
$$

where $\left[\mathbf{G}_{s}\left(r_{n}, \gamma_{n}\right)\right]_{n, m}=\sqrt{2 \pi} j^{m} J_{m}\left(\kappa r_{n}\right) e^{j m \gamma_{n}}$ defines the $(n, m)$ th element of the sampling matrix $\mathbf{G}_{s}\left(r_{n}, \gamma_{n}\right)$ and, $J_{m}(\cdot)$ is the Bessel function of the first kind of order $m$. Note, $\mathbf{G}_{s}\left(r_{n}, \gamma_{n}\right)$ depends on the array configuration only, and it is independent from the wavefield (in particular from $\phi$ ).

The idea of wavefield modeling is to write the signal-dependent part of the array output in (1) as the product of a sampling matrix $\mathbf{G}_{s}$ (independent from the wavefield) and a coefficient vector $\mathbf{d}_{s}(\phi)$ (independent from the array) [13], [14]. Consequently, by writing (3) in matrix form, we can express the key concept of MST by

$$
\mathbf{b}(\phi)=\mathbf{G}_{s} \mathbf{d}_{s}(\phi) \quad \text { for } \phi \in[-\pi, \pi) .
$$

In case of 1-D (e.g., azimuth) estimation, the $m$ th component of $\mathbf{d}_{s}(\phi)$ is

$$
\left[\mathbf{d}_{s}(\phi)\right]_{m}=\frac{1}{\sqrt{2 \pi}} e^{-j m \phi}, \quad \text { for } m=\ldots,-1,0,1, \ldots
$$

For the 2-D (azimuth and elevation) estimation problem, $\mathbf{d}_{s}(\phi, \theta)$ may be expressed using spherical harmonics [13], [22].

Note that for a given antenna array, the MST can perfectly model the array steering vector $\mathbf{b}(\phi)$ for $\phi \in[-\pi, \pi)$ only by using an infinite ${ }^{1}$ number of Fourier coefficients [see (4) and (5)]. However, this condition can be relaxed. In practice, the sampling matrix may be truncated by considering only a finite number of excitation modes $m$ [9], [10]. Ideally, the resulting truncation error can be made arbitrarily small, only by increasing the number of modes.

\footnotetext{
${ }^{1}$ In [13], the sampling matrix is introduced as an operator, which operates on functions belonging to an infinite dimensional Hilbert space $\mathcal{H}$ so that $\mathbf{G}_{s}$ : $\mathcal{H} \rightarrow \mathbb{C}^{N \times 1}$. The operator $\mathbf{G}_{s}$ can also be interpreted as a matrix with $N$ rows and an infinite number of columns.
} 
For example, in [12], [13], and [16], it is proposed to truncate the coefficient vector $\mathbf{d}_{s}(\phi)$ at $|m| \approx 2 \kappa R$, where $R$ is the radius of the smallest circle centered at the origin of the array and enclosing all the physical components. In Section VI-A-1), we propose a different criterion based on practical observations. In order to minimize the modeling error, a larger number of excitation modes $m$ should be selected [10]. This leads to a smaller array manifold reconstruction error and, consequently, to a smaller error in the DoA estimates.

\section{EFFECTIVE Aperture Distribution FunCTION}

A practical approach for the determination of the sampling matrix $\mathbf{G}_{s}$ starting from the calibration measurement of an antenna array is here presented. For simplicity, we restrict the following discussion to arrays formed by uncoupled and omnidirectional sensors, and we assume noise-free calibration data. However, this does not limit the generality of the discussion.

The response of an antenna array to a far-field source can be modeled by measuring the directional characteristic of the array in an anechoic chamber. Let us define the set of calibration points $\phi^{\text {cal }}=\left\{\phi_{1}^{\text {cal }}, \phi_{2}^{\text {cal }}, \ldots, \phi_{Q}^{\text {cal }}\right\}$, where $Q(Q \gg N)$ is the number of calibration points.

We may measure the array response to a far-field source $\mathbf{b}^{\text {cal }}(\phi) \in \mathbb{C}^{N \times 1}$ by moving the source around the array at a fixed co-elevation angle, e.g., at $\theta=90^{\circ}$ in the azimuthal range $\phi \in[-\pi, \pi)$. Alternatively, the same result can be obtained by fixing the source location and rotating the array about its centroid. This creates a discrete set of measured points (along $\phi)$, which represents a discrete $2 \pi$-periodic function in $\phi$.

Combining the measurements of an $N$ elements antenna array into a matrix, we can form the calibration matrix $\mathbf{B}_{\phi}^{\text {cal }}=\left[\mathbf{b}^{\text {cal }}\left(\phi_{1}\right), \ldots, \mathbf{b}^{\text {cal }}\left(\phi_{Q}\right)\right] \in \mathbb{C}^{N \times Q}$. Note that $\mathbf{B}_{\phi}^{\text {cal }}$ is assumed to have full row-rank if $\phi_{k}^{\text {cal }} \neq \phi_{q}^{\text {cal }}$ for $k \neq q$.

By computing the $Q$-point inverse discrete Fourier transform (IDFT) of the $2 \pi$-periodic discrete set of measured points and using (3), we can express the $n$th row of $\mathbf{B}_{\phi}^{\text {cal }}$ as

$$
\begin{aligned}
\frac{1}{Q} \sum_{q=1}^{Q} e^{j \kappa r_{n} \cos \left(\gamma_{n}-\phi_{q}\right)} e^{j m \phi_{q}}=j^{m} J_{m}\left(\kappa r_{n}\right) e^{j m \gamma_{n}} \\
+\sum_{l=1}^{\infty}\left(j^{g} J_{g}\left(\kappa r_{n}\right) e^{j g \gamma_{n}}+j^{h} J_{h}\left(\kappa r_{n}\right) e^{j h \gamma_{n}}\right)
\end{aligned}
$$

where $m=\ldots,-1,0,1, \ldots$ with $g=Q l-m$ and $h=Q l+m$. Expression (6) describes a $Q$-periodic signal comprised of two terms. The first term defines the $(n, m)$ th element of the sampling matrix $\left[\mathbf{G}_{s}\left(r_{n}, \gamma_{n}\right)\right]_{n, m}$ in (3). The second term describes the set of, infinite many, replicas of the first term due to the angular sampling of the array manifold during calibration. The $l$ th replica is shifted by $Q l$ from the principal term (at $l=0$ ).

From (6), we observe that since $J_{-m}(\cdot)=(-1)^{m} J_{m}(\cdot)$, the magnitude of the sampling matrix $\left[\mathbf{G}_{s}\left(r_{n}, \gamma_{n}\right)\right]$ (the principal term) is an even function of $m$. Moreover, as described in [13], its magnitude decays superexponentially as the number of modes $|m| \rightarrow \infty$. Consequently, when the number of calibration points $Q$ is chosen sufficiently large $(Q \gg N)$, the replicas are far apart, and the aliasing in the area of interest is negligible.
Considering the $Q$-points periodicity of (6), we define the EADF matrix $\mathbf{G}_{e} \in \mathbb{C}^{N \times Q}$ as

$$
\left[\mathbf{G}_{e}\right]_{n, m}=\left[\mathbf{G}_{s}\right]_{n, m}
$$

for $n=0, \ldots, N-1$ and $m \in[-((Q-1) / 2),((Q-1) / 2)]$. The variable $m$ denotes the excitation mode index of the EADF. Consequently, we can rewrite (4) as

$$
\mathbf{b}(\phi)=\mathbf{G}_{e} \mathbf{d}_{e}(\phi)+\varepsilon_{a}(Q) \text { for } \phi \in[-\pi, \pi)
$$

where $\varepsilon_{a}(Q) \in \mathbb{C}^{N \times 1}$ contains the modeling error due to aliasing and $\left[\mathbf{d}_{e}(\phi)\right]_{m}=e^{-j m \phi} / \sqrt{2 \pi}$. Observe, as $Q \rightarrow \infty$, the $L_{2}$ norm of the aliasing error $\left\|\varepsilon_{a}(Q)\right\|^{2} \rightarrow 0$. Practically, for a sufficiently large $Q$, the matrices $\mathbf{G}_{s}$ and $\mathbf{G}_{e}$ carry the same information [13], [18], [21].

Due to the superexponential decay of the magnitude of the sampling matrix [13], the contribution of modes with a sufficiently high index $|m|$ (within a period) to the array manifold reconstruction is negligible. Hence, a truncated version $\mathbf{G} \in \mathbb{C}^{N \times M}$ of the EADF matrix $\mathbf{G}_{e}$ can be defined as

$$
[\mathbf{G}]_{n, m}=\left[\mathbf{G}_{e}\right]_{n, m}
$$

for $n=0, \ldots, N-1$ and $m \in\left[-M_{e}, M_{e}\right]$, where $N<M<Q$ and $M=2 M_{e}+1$.

As a result, we can rewrite (3) using a finite number of excitation modes $M$ (Fourier coefficients) as

$$
\begin{aligned}
e^{j \kappa r_{n} \cos \left(\gamma_{n}-\phi\right)}= & \frac{1}{\sqrt{M}} \sum_{m=-M_{e}}^{M_{e}}[\mathbf{G}]_{n, m} e^{-j m \phi}+[\varepsilon(M, Q)]_{n} \\
= & \sum_{m=-M_{e}}^{M_{e}} j^{m} J_{m}\left(\kappa r_{n}\right) e^{j m \gamma_{n}} e^{-j m \phi} \\
& +[\varepsilon(M, Q)]_{n}
\end{aligned}
$$

where $\varepsilon(M, Q)=\varepsilon_{a}(Q)+\varepsilon_{t}(M) \in \mathbb{C}^{N \times 1}$ contains the modeling error due to aliasing $\varepsilon_{a}(Q)$ and truncation $\varepsilon_{t}(M)$. Combining (4), (8), and (10), we rewrite the MST principle

$$
\mathbf{b}(\phi)=\mathbf{G d}(\phi)+\varepsilon(M, Q) \quad \text { for } \phi \in[-\pi, \pi)
$$

where the Vandermonde vector $\mathbf{d}(\phi) \in \mathbb{C}^{M \times 1}$ is of the form

$$
\mathbf{d}(\phi)=\frac{1}{\sqrt{M}}\left[e^{j \frac{M-1}{2} \phi}, \ldots, 1, \ldots, e^{-j \frac{M-1}{2} \phi}\right]^{T} .
$$

Observe that for sufficiently large $Q$, the error due to truncation dominates, i.e., $\left\|\varepsilon_{a}\right\| \ll\left\|\varepsilon_{t}\right\|$. In the following, we assume that a sufficiently large number of calibration points have been measured, such that $\varepsilon(M, Q) \approx \varepsilon_{t}(M) \triangleq \varepsilon(M)$. For more detail and numerical evaluation of the error, see [10] and [13].

However, this does not apply in a real-world scenario where the calibration data always contain measurement noise and $M$ cannot be arbitrarily chosen. In Fig. 1, we compare the EADFs computed from simulated calibration measurements with and without noise. For noise-free calibration data, the error floor is determined by the computational accuracy of the used computer (IEEE-754, 64-bit float). Otherwise, the perturbed EADF (computed with noisy calibration data) saturates at the measurement noise error level. Clearly, there is a link between the calibration 


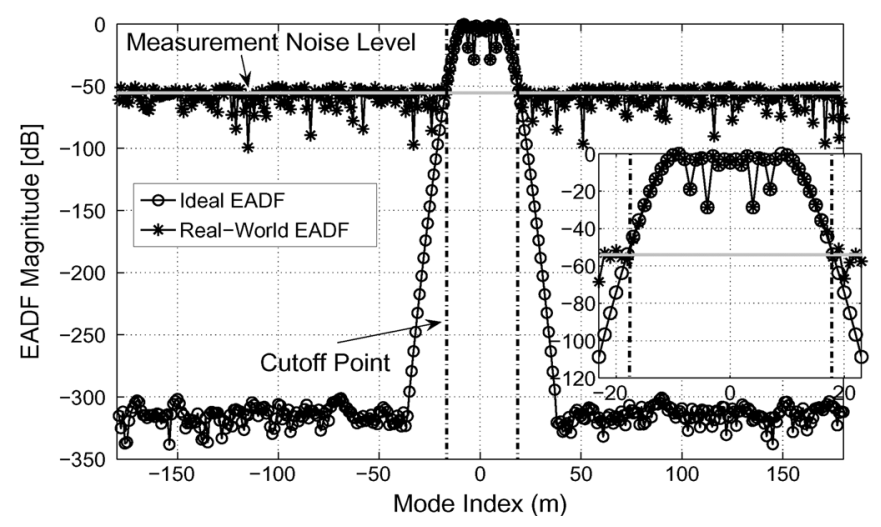

Fig. 1. Ideal and real-world EADFs computed from $Q=360$ simulated and calibration points, respectively. The measurement noise level $\sigma_{W}^{2}$ limits the maximum number of usable modes $M$. The truncated EADF is within the truncation boundaries.

noise power and $M$. In Section VI-A-1), we present a criterion for selecting the optimum number of modes $M$ for a given calibration signal-to-noise ratio (SNR).

The MST expression in (11) has a useful property. It can be algebraically differentiated. In fact, since the EADF vector $\mathbf{g}_{n}$ ( $n$th row of $\mathbf{G}$ ) is independent from the unknown angular parameter $\phi[13]$, the derivative of the $n$th sensor beampattern with respect to $\phi$ can be expressed as [15], [17], [18]

$$
\delta_{n}(\phi)=\frac{\partial b_{n}(\phi)}{\partial \phi}=\mathbf{g}_{n} \frac{\partial \mathbf{d}(\phi)}{\partial \phi} .
$$

This will be exploited in Section VII.

\section{A. Discussion}

Let us consider the theoretical case of noise-free calibration data of a real-world array. Every sensor has a unique beampattern that is influenced by the neighboring sensors, i.e., mutual coupling. Performing calibration measurements of the whole antenna array implies that the $n$th measured beampattern is influenced by the other array elements. Moreover, the calibration data contains also array nonidealities, such as antenna manufacturing errors, and orientation and position of the sensors. A comparison between the EADF of an ideal and real-world array can be found in [8].

In (7) and (9), the matrices $\mathbf{G}_{s}, \mathbf{G}_{e}$, and $\mathbf{G}$ have the same number of rows $N$ but different number of columns, i.e., $\infty, Q$ or $M(M<Q)$, respectively. The EADF and truncated EADF matrices represent a reduced version of the sampling matrix.

Let us consider the $n$th row of each of the above matrices, defined by $\left[\mathbf{G}_{s}\right]_{n},\left[\mathbf{G}_{e}\right]_{n}$, and $[\mathbf{G}]_{n}$, respectively. $\left[\mathbf{G}_{s}\right]_{n}$ is an aperiodic discrete function having an infinite support, $\left[\mathbf{G}_{e}\right]_{n}$ can be understood as a $Q$-periodic discrete function and, within a period, $[\mathbf{G}]_{n}$ is an $M$-points truncated version of $\left[\mathbf{G}_{e}\right]_{n}$. The sampling matrix $\mathbf{G}_{s}$ contains the full description of the array. By choosing $Q$ and $M$ properly, the matrices $\mathbf{G}_{e}$ and $\mathbf{G}$ also contain a sufficiently accurate description of the antenna array under investigation.

\section{ELEMENT-SPACE ROOT-MUSIC}

Here, we briefly present the ES-root-MUSIC algorithm. It allows azimuthal DoA estimation of noncoherent sources at a fixed elevation angle on arbitrary array configurations [9], [10]. The algorithm does not require interpolation or mapping of the array data matrix. Assuming the true array manifold is known, for uncorrelated sources and no finite sample effects, it is an unbiased estimator [24]-[26]. This motivates the use of ES-rootMUSIC in order to study the impact of the calibration measurement noise on the DoA estimation; see Section VI.

It is important to remember that in a real-world radio systems, the maximum SNR is mainly limited by the transmit power and the receiver noise. Whenever the SNR $\ll \min _{\phi}(\|\mathbf{b}(\phi)\|) /\|\varepsilon(M)\|$, the residual modeling error can be neglected and (11) still holds. In other words, if the error floor caused by $\varepsilon(M)$ is much smaller than the variance of the DoA estimates at the highest achievable SNR, the modeling error $\varepsilon(M)$ can be neglected.

Therefore, combining (1) and (11), we can write

$$
\mathbf{X}=\mathbf{B S}+\mathbf{N}=\mathbf{G D S}+\mathbf{N}
$$

where $\mathbf{D} \in \mathbb{C}^{M \times P}$ is a matrix formed as $\mathbf{D}=\left[\mathbf{d}\left(\phi_{1}\right), \ldots\right.$, $\left.\mathbf{d}\left(\phi_{P}\right)\right]$, and $\phi_{1}, \ldots, \phi_{P}$ are the true DoAs of the $P$ sources. Here, $\mathbf{G} \in \mathbb{C}^{N \times M}$ is the truncated EADF matrix defined in Section IV.

The element-space covariance matrix can be expressed by

$$
\mathbf{R}_{x}=\mathbf{E}_{s} \boldsymbol{\Lambda}_{s} \mathbf{E}_{s}^{H}+\sigma_{n}^{2} \mathbf{E}_{n} \mathbf{E}_{n}^{H}=\mathbf{G D R}_{s} \mathbf{D}^{H} \mathbf{G}^{H}+\sigma_{n}^{2} \mathbf{I}
$$

where $\mathbf{I}$ is the $N \times N$ identity matrix, $\mathbf{R}_{s}$ is the $P \times P$ signal covariance matrix, and the matrices of eigenvectors $\mathbf{E}_{s}$ and $\mathbf{E}_{n}$ span the $N \times P$ signal and the $N \times(N-P)$ noise subspaces, respectively. The key idea used in the derivation of ES-rootMUSIC is that $\mathbf{E}_{s}, \mathbf{B}$, and GD span the same subspace. Consequently, GD is orthogonal to $\mathbf{E}_{n}$ [10].

Exploiting MST, we can express the pseudospectrum of element-space MUSIC for arrays of arbitrary geometry as [10]

$$
S_{\text {music }}(\phi)=\left(\mathbf{d}^{H}(\phi) \mathbf{G}^{H} \mathbf{E}_{n} \mathbf{E}_{n}^{H} \mathbf{G d}(\phi)\right)^{-1}
$$

which allows applying fast polynomial rooting algorithms instead of exhaustive search, e.g., root-MUSIC [1], [25]. By substituting $z=e^{j \phi}$ into the $M \times 1$ Vandermonde structured vector $\mathbf{d}(\phi),(16)$ can be rewritten in polynomial form.

The azimuth estimates are computed from the argument of the $P$ roots $z_{p}$ closest to the unit circle, i.e., $\hat{\phi}_{p}=\angle\left(z_{p}\right)$. A summary of the ES-root-MUSIC algorithm is given in Table I. Note, the matrix $\mathbf{G}$ is formed offline, and it needs to be computed only once for a given antenna array.

So far, we have assumed a large number of observations $\left(\mathbf{R}_{x}, \mathbf{E}_{s}\right.$, and $\mathbf{E}_{n}$ are known) and noise-free calibration data. In the next section, we study the effect of modeling error due to noisy calibration data on the DoA estimates, i.e., when the orthogonality between $\mathbf{E}_{n}$ and $\mathbf{b}(\phi)$ is impaired.

\section{EADF PERTURBATION IMPACT ON DOA ESTIMATES}

In this section, we focus on the MST when an estimated EADF computed from noisy calibration data is used. In par- 
TABLE I

ES-ROOT-MUSIC ALGORITHM

- Computation of the truncated EADF matrix $\mathbf{G}$ for arbitrary arrays configuration (offline process):

i) form the calibration matrix $\mathbf{B}_{\phi}^{\text {cal }} \in \mathbb{C}^{N \times Q}$,

ii) compute the EADF matrix $\mathbf{G}_{e} \in \mathbb{C}^{N \times Q}$ as the $Q$-points IDFT of $\mathbf{B}_{\phi}^{\text {cal }}$,

iii) form the truncated EADF matrix $\mathbf{G} \in \mathbb{C}^{N \times M}$ by selecting the $M$ middle columns of $\mathbf{G}_{e}$ as

- $M=\{\mu \in \mathbb{N}: N<\mu \ll Q\}$ for noise-free calibration measurement $(\mathbf{W}=0)$

- $M=\left\{\mu \in \mathbb{N}: N<\mu \leq M_{\eta} \ll Q, \mu=M_{\eta}\right\}$ for noisy calibration measurement $(\mathbf{W} \neq 0)$

- Perform angular estimation (online process):

1) form the ES array data matrix $\mathbf{X}$ and the ES noise subspace $\mathbf{E}_{\eta}$ as in equations (1) and (15),

2) apply the root-MUSIC algorithm on expression (16).

ticular, we investigate the impact of the noise recorded during the array calibration measurement on the DoA estimates. We assume that the angular estimates are found by the ES-rootMUSIC algorithm; see Section V.

Let us model the perturbed EADF $\widetilde{\mathbf{G}}_{e} \in \mathbb{C}^{N \times Q}$ as

$$
\widetilde{\mathbf{G}}_{e}=\mathbf{G}_{e}+\mathbf{W}_{e}
$$

where $\mathbf{G}_{e} \in \mathbb{C}^{N \times Q}$ is the ideal (calibration error-free) EADF matrix, and $\mathbf{W}_{e} \in \mathbb{C}^{N \times Q}$ contains the calibration measurement noise. The noise is modeled as a zero-mean, independent and identically distributed (i.i.d.) circular complex Gaussian process with variance $\sigma_{W}^{2}$.

Combining (8), (11), and (17), and considering only the modeling error due to a truncation down to $M$ modes, we can express the perturbed array steering vector as

$$
\widetilde{\mathbf{b}}(\phi, \mathbf{W}, M)=\widetilde{\mathbf{G}} \mathbf{d}+\varepsilon(M)=\mathbf{G} \mathbf{d}+\mathbf{W} \mathbf{d}+\varepsilon(M)
$$

where $\mathbf{W}$ is an $N \times M$ block of the matrix $\mathbf{W}_{e}$. Equation (18) is comprised of three terms. The first is the ideal MST using a calibration noise-free EADF matrix. The quantities $\mathbf{W d}$ and $\varepsilon(M)$ represent error terms depending on the calibration noise and the truncation error, respectively.

In the next sections, we investigate the impact of these error terms on both the EADF and the MSE of the DoA estimates. Illustrative examples are here presented.

\section{A. Dependence on the Truncation Error}

Let us fix the calibration noise power $\sigma_{W}^{2}$ and focus on the effects due to truncation error. This situation is close to reality, particularly when only one set of calibration measurements is available and the system designer has to choose the optimum number for $M$.

In Fig. 2(a), we depict an ideal (noise-free) and perturbed (noisy) EADF. Here, we also plot three possible choices of selecting the number of modes $M$. For the simulation, we have used an uniform circular array (UCA) with $N=8$ sensors, radius $r=0.5 \lambda$, and EADF-to-calibration noise ratio (ENR) $=$ $80 \mathrm{~dB}$. The ENR is defined as ENR $=\mathcal{P}_{E} / \mathcal{P}_{\eta}$, where $\mathcal{P}_{E}$ and $\mathcal{P}_{\eta}$ define the true EADF $\mathbf{G}_{e}$ and calibration noise powers for the $n$th sensor; see also Section VI-A-2).

In Fig. 2(b), we show the impact of $M$ on the root meansquare error (RMSE) of the estimates. For a fixed value of the ENR, each curve has a minimum representing the minimum

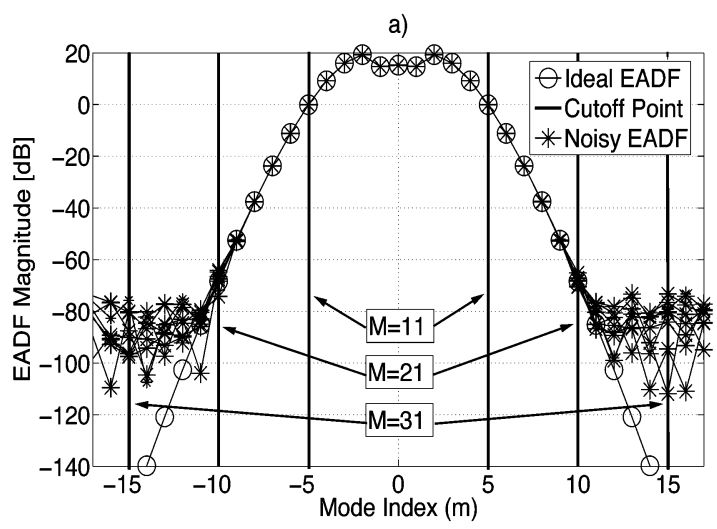

b)

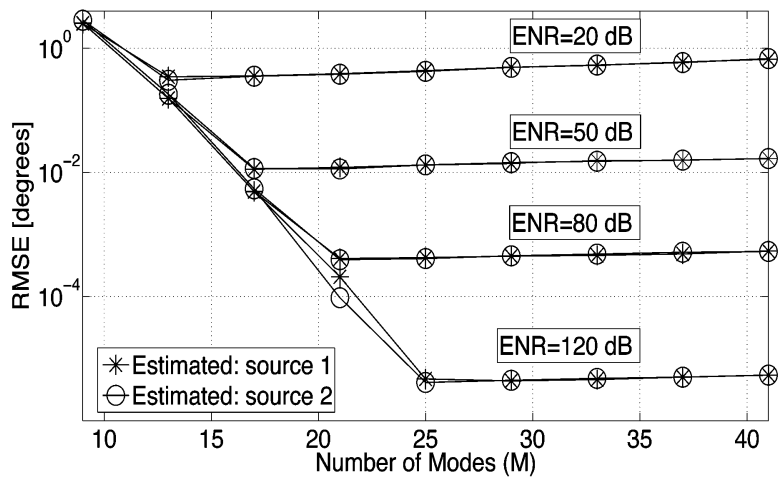

Fig. 2. In (a), different truncation boundaries for $\widetilde{\mathbf{G}}_{e}$ are depicted. When $M=$ 11 (too small), useful information is discarded. If $M=31$ (too large), we consider modes which are corrupted by calibration noise. In (b), the estimated RMSE using ES-root-MUSIC is shown. For a fixed value of ENR, by increasing $M$, the error decreases to a minimum value. Hence, by further increasing $M$, the RMSE slowly increases due to the calibration noise in the EADF.

achievable error in that scenario, e.g., $M=21$ for ENR $=$ $80 \mathrm{~dB}$. Consequently, there is an optimum number of modes $M_{\eta}$, which minimizes the antenna modeling error. For these simulations, we used two uncorrelated sources at $\left(\phi_{1}, \phi_{2}\right)=$ $\left(25^{\circ}, 45^{\circ}\right)$, observation noise-free data, an $N=8$ elements UCA with $r=0.5 \lambda$, and 1500 independent realizations of $\mathbf{W}$.

1) Selection of the Number of Modes: As described in [10], ideally $M$ can be selected such that $N \leq M \leq Q$. However, due to calibration noise, we have a smaller selection range $N \leq$ $M \leq M_{\eta} \leq Q$, where $|m|=\left(M_{\eta}-1\right) / 2$ is the highest mode order having a magnitude larger than the calibration noise variance $\sigma_{W}^{2}$; see also Table I. 
Given a perturbed EADF computed from noisy calibration data, the following rule of thumb can be used: the optimal number of modes $M_{\eta}$, which minimize the modeling error, is given by the number of modes having a magnitude larger than the calibration noise level. This criterion is also confirmed by the following observations.

Choosing $M>M_{\eta}$ leads to a gradual increase of the RMSE, because those additional modes are severely degraded by calibration noise. This also means that the term $\mathbf{W d}$ dominates over $\varepsilon(M)$; see (18). Conversely, by selecting $M<M_{\eta}$, the RMSE increases due to truncation error.

2) Advantages of Truncation: Truncating the perturbed EADF $\widetilde{\mathbf{G}}_{e}$ to $\widetilde{\mathbf{G}}$ having $M=M_{\eta}$ modes has two main effects. First, it reduces the amount of data that we have to store [17]. Second, it improves the accuracy of the array data model approximately by a factor of $10 \log _{10}(Q / M)$ in ENR.

In fact, by using Parseval's theorem, we can write the ENR of the perturbed EADF $\widetilde{\mathbf{G}}_{e}$ for the $n$th sensor as

$$
\mathrm{ENR}_{\widetilde{\mathbf{G}}_{e}}=10 \log _{10}\left(\frac{\sum_{i=1}^{Q}\left|\left[\mathbf{G}_{e}\right]_{n, i}\right|^{2}}{\sigma_{W}^{2} Q}\right)
$$

where $\left[\mathbf{G}_{e}\right]_{n, i}$ is the $(n, i)$ th element of the true EADF matrix. Furthermore, since the magnitude of the ideal EADF $\mathbf{G}_{e}$ decays superexponentially for modes in the range $|m| \in(M, Q)$ [13], we may truncate the ideal EADF at $M<Q$ modes and approximate $\sum_{i=1}^{Q}\left|\left[\mathbf{G}_{e}\right]_{n, i}\right|^{2} \approx \sum_{i=1}^{M}\left|[\mathbf{G}]_{n, i}\right|^{2}$. Therefore, the ENR of the truncated perturbed EADF $\widetilde{\mathbf{G}}$ is

$$
\begin{aligned}
\operatorname{ENR}_{\widetilde{\mathbf{G}}} & =10 \log _{10}\left(\frac{\sum_{i=1}^{M}\left|[\mathbf{G}]_{n, i}\right|^{2}}{\sigma_{W}^{2} M}\right) \\
& \approx \operatorname{ENR}_{\widetilde{\mathbf{G}}_{e}}\left(10 \log _{10}\left(\frac{Q}{M}\right)\right) .
\end{aligned}
$$

For a fixed number of selected modes $M$, the larger is the number of calibration points $Q$, the higher ENR we get. This is an advantage of MST/EADF-based techniques over methods that perform array manifold interpolation directly using the calibration data.

\section{B. Dependence on the Calibration Noise}

Now, we consider to fix the truncation error level (by selecting $M$ ), and we investigate the impact of the calibration noise power on the RMSE of the DoA estimates.

In Fig. 3(a), we depict an ideal (noise-free) and perturbed (noisy) EADF in four different ENR scenarios. Let us focus on the mode indexes $m \in[-10,10]$, i.e., $M=21$. Clearly, the impact of the calibration noise on the considered modes reduces as the ENR increases.

In Fig. 3(b), we show the impact of the calibration noise on the DoA estimates. By fixing $M$ and increasing the ENR, we can observe that the RMSE decreases until it reaches an error floor. For example, for $M=17$, the curve saturates at ENR $=60 \mathrm{~dB}$. This means that for an ENR $<60 \mathrm{~dB}$, the term Wd dominates over the truncation error. On the other hand, for ENR $>60 \mathrm{~dB}$, the term $\varepsilon(M)$ dominates and, since $M$ is fixed, the error on the DoA estimates remains constant. The following settings have been used in the simulation: UCA with $N=8$, radius $r=0.5 \lambda$,
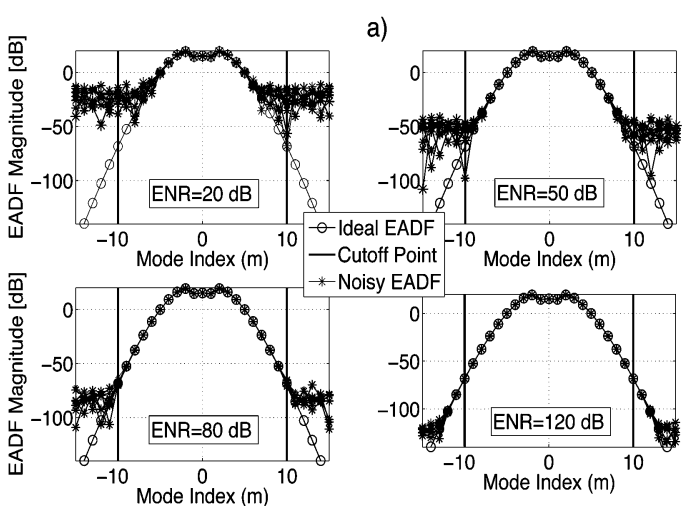

b)

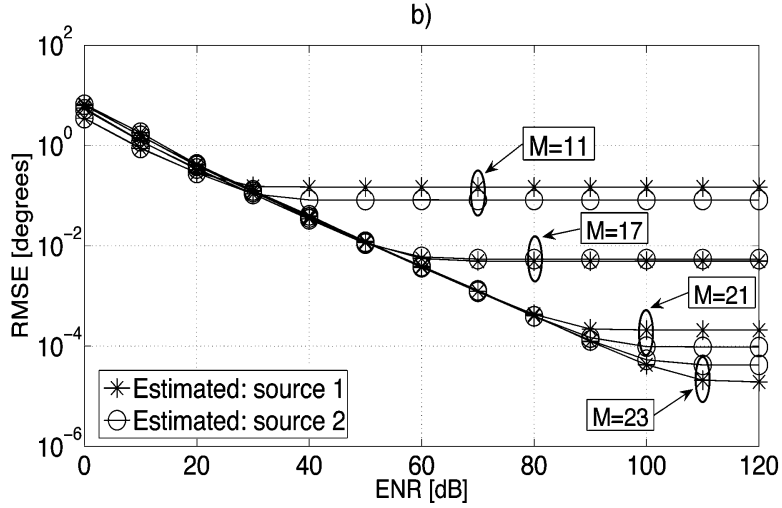

Fig. 3. In (a), comparison between the ideal (noise-free) and real-world (noisy) EADFs. By increasing the ENR, the influence of the calibration noise on the $M=21$ selected modes fades. In (b), the estimated RMSE using ES-rootMUSIC is shown. The error decreases as the calibration noise decreases (and ENR increases) until it reaches a saturation floor. The error floor depends on $M$ through the truncation error, and it decreases as $M$ increases.

$\left(\phi_{1}, \phi_{2}\right)=\left(25^{\circ}, 45^{\circ}\right)$, observation noise-free data, and 1500 independent Monte Carlo trials.

\section{ERROR ANALYSIS}

Let us assume the calibration noise model defined in Section VI. Here, we present an analysis of the error in the DoA estimates due to calibration noise and truncation. Note that the following analysis is carried out under the assumption of noise-free observations.

For the analysis, we use ES-root-MUSIC [9], [10]. The estimates are given by the $P$ smallest local minima of the quadratic expression

$$
\tilde{f}(\phi)=\mathbf{d}^{H}(\phi) \widetilde{\mathbf{G}}^{H} \mathbf{E}_{n} \mathbf{E}_{n}^{H} \widetilde{\mathbf{G}} \mathbf{d}(\phi)
$$

where $\mathbf{E}_{n} \in \mathbb{C}^{N \times(N-P)}$ spans the true noise subspace, $\widetilde{\mathbf{G}} \in \mathbb{C}^{N \times M}$ is a truncated version of the estimated EADF, and $\mathbf{d}(\phi) \in \mathbb{C}^{M \times 1}$ is the Vandermonde structured vector in (12), which depends on the unknown parameter $\phi$. For a discussion of large sample properties of MUSIC and root-MUSIC, see [25]-[27].

Note that, in order to simplify the notation, we will write $\mathbf{d}(\phi)$ as $\mathbf{d}_{\phi}$ and $\mathbf{d}\left(\phi_{i}\right)$ as $\mathbf{d}_{i}$ from now on.

\section{A. Single Calibration Measurement}

Let us construct the calibration matrix $\mathbf{B}_{\phi}^{\text {cal }} \in \mathbb{C}^{N \times Q}$ by measuring a given antenna array. We form the matrix $\overline{\mathbf{G}}=\mathbf{G}+$ 
$\overline{\mathbf{W}} \in \mathbb{C}^{N \times M_{\eta}}$ by truncating the IDFT of the calibration data $\overline{\mathbf{G}}_{e} \in \mathbb{C}^{N \times Q}$ down to $M=M_{\eta}$ modes; see Section VI-A-1). Observe that $\overline{\mathbf{G}}_{e}, \overline{\mathbf{G}}$, and $\overline{\mathbf{b}}\left(\phi, \overline{\mathbf{W}}, M_{\eta}\right)$ depend on a particular realization of the calibration noise $\overline{\mathbf{W}}$.

Rewriting (21) as $\bar{f}(\phi)=\mathbf{d}_{\phi}^{H} \overline{\mathbf{G}}^{H} \mathbf{E}_{n} \mathbf{E}_{n}^{H} \overline{\mathbf{G}} \mathbf{d}_{\phi}$, we can find an expression for the error caused by $\overline{\mathbf{W}}$. We expand the firstorder derivative of $\bar{f}(\phi)$ with respect to $\phi_{i}$ and evaluate it at $\hat{\phi}_{i}$. For small enough errors, we can write [7], [24]

$$
0=\bar{f}^{\prime}\left(\hat{\phi}_{i}\right) \approx \bar{f}^{\prime}\left(\phi_{i}\right)+\bar{f}^{\prime \prime}\left(\phi_{i}\right)\left(\hat{\phi}_{i}-\phi_{i}\right)
$$

where $\phi_{i}$ and $\hat{\phi}_{i}$ are the true and estimated DoA for the $i$ th source, respectively, and $\left.\bar{f}^{\prime}\left(\hat{\phi}_{i}\right) \triangleq(\partial \bar{f}(\phi) / \partial \phi)\right|_{\phi=\hat{\phi}_{i}}$. Moreover, let us define the $M \times 1$ vector $\mathbf{d}_{\phi}^{\prime}$ as

$\mathbf{d}_{\phi}^{\prime} \triangleq \frac{\partial \mathbf{d}_{\phi}}{\partial \phi}=\operatorname{diag}\left\{j \frac{(M-1)}{2}, \ldots, 0, \ldots,-j \frac{(M-1)}{2}\right\} \mathbf{d}_{\phi}$

and, similarly, $\mathbf{d}_{\phi}^{\prime \prime} \triangleq\left(\partial \mathbf{d}_{\phi}^{\prime} / \partial \phi\right)$. Here, $\operatorname{diag}\{\cdot\}$ denotes a diagonal matrix formed from the elements within the brackets.

The first derivative of $\bar{f}(\phi)$ in (21) with respect to $\phi$ is

$$
\begin{aligned}
\bar{f}^{\prime}(\phi) & =\mathbf{d}_{\phi}^{\prime H} \overline{\mathbf{G}}^{H} \mathbf{E}_{n} \mathbf{E}_{n}^{H} \overline{\mathbf{G}} \mathbf{d}_{\phi}+\mathbf{d}_{\phi}^{H} \overline{\mathbf{G}}^{H} \mathbf{E}_{n} \mathbf{E}_{n}^{H} \overline{\mathbf{G}} \mathbf{d}_{\phi}^{\prime} \\
& =2 \Re\left\{\mathbf{d}_{\phi}^{\prime H} \overline{\mathbf{G}}^{H} \mathbf{E}_{n} \mathbf{E}_{n}^{H} \overline{\mathbf{G}} \mathbf{d}_{\phi}\right\}
\end{aligned}
$$

where $\Re\{\cdot\}$ denotes the real part of the expression within the brackets. Similarly, the second derivative $\bar{f}^{\prime \prime}(\phi)=$ $\left(\partial \bar{f}^{\prime}(\phi) / \partial \phi\right)$ is

$\bar{f}^{\prime \prime}(\phi)=2 \Re\left\{\mathbf{d}_{\phi}^{\prime \prime H} \overline{\mathbf{G}}^{H} \mathbf{E}_{n} \mathbf{E}_{n}^{H} \overline{\mathbf{G}} \mathbf{d}_{\phi}+\mathbf{d}_{\phi}^{\prime H} \overline{\mathbf{G}}^{H} \mathbf{E}_{n} \mathbf{E}_{n}^{H} \overline{\mathbf{G}} \mathbf{d}_{\phi}^{\prime}\right\}$.

Combining (22), (24), and (25), we can establish a relationship between the errors in the calibration data and in the DoA estimates, when MUSIC is used. A closed-form expression for the error at $\phi_{i}$ can be written as in

$$
\begin{aligned}
& \left(\hat{\phi}_{i}-\phi_{i}\right) \\
& \approx-\frac{\Re\left\{\mathbf{d}_{i}^{\prime H} \overline{\mathbf{G}}^{H} \mathbf{E}_{n} \mathbf{E}_{n}^{H} \overline{\mathbf{G}} \mathbf{d}_{i}\right\}}{\Re\left\{\mathbf{d}_{i}^{\prime \prime} \overline{\mathbf{G}}^{H} \mathbf{E}_{n} \mathbf{E}_{n}^{H} \overline{\mathbf{G}} \mathbf{d}_{i}+\mathbf{d}_{i}^{\prime H} \overline{\mathbf{G}}^{H} \mathbf{E}_{n} \mathbf{E}_{n}^{H} \overline{\mathbf{G}} \mathbf{d}_{i}^{\prime}\right\}} .
\end{aligned}
$$

By observing (26), the following consideration can be made.

a) The term $\mathbf{E}_{n}^{H} \overline{\mathbf{G}} \mathbf{d}_{i}$ is a measure of the orthogonality between the true noise subspace $\mathbf{E}_{n}$ and the modeled array steering vector $\overline{\mathbf{G}} \mathbf{d}_{i}$; see (18). For a fixed $M_{\eta}$, increasing the ENR causes $\overline{\mathbf{G}} \rightarrow \mathbf{G}$ and $\left\|\mathbf{E}_{n}^{H} \mathbf{G d}_{i}\right\|^{2} \rightarrow \epsilon\left(M_{\eta}\right)$. The quantity $\epsilon\left(M_{\eta}\right)$ represents the error in orthogonality and it defines the error floor as depicted in Fig. 3. Then, by selecting $M>M_{\eta}, \mathbf{G} \rightarrow \mathbf{G}_{e}$, and $\epsilon(M) \rightarrow 0$. As a result the following expression holds:

$$
\lim _{\mathrm{ENR} \rightarrow \infty, M \rightarrow \infty}\left\|\mathbf{E}_{n}^{H} \overline{\mathbf{G}} \mathbf{d}_{i}\right\|^{2}=\left\|\mathbf{E}_{n}^{H} \mathbf{G}_{e} \mathbf{d}_{i}\right\|^{2} \approx 0 .
$$

This also ensures that the modeling error caused by using MST is zero if a perfect model for the antenna array is used.

b) For the term $\mathbf{d}_{i}^{\prime H} \overline{\mathbf{G}}^{H} \mathbf{E}_{n}$, we can observe that

$$
\lim _{\mathrm{ENR} \rightarrow \infty, M \rightarrow \infty}\left\|\mathbf{d}_{i}^{\prime H} \overline{\mathbf{G}}^{H} \mathbf{E}_{n}\right\|^{2}=\left\|\mathbf{d}_{i}^{\prime H} \mathbf{G}_{e}^{H} \mathbf{E}_{n}\right\|^{2} \text {. }
$$

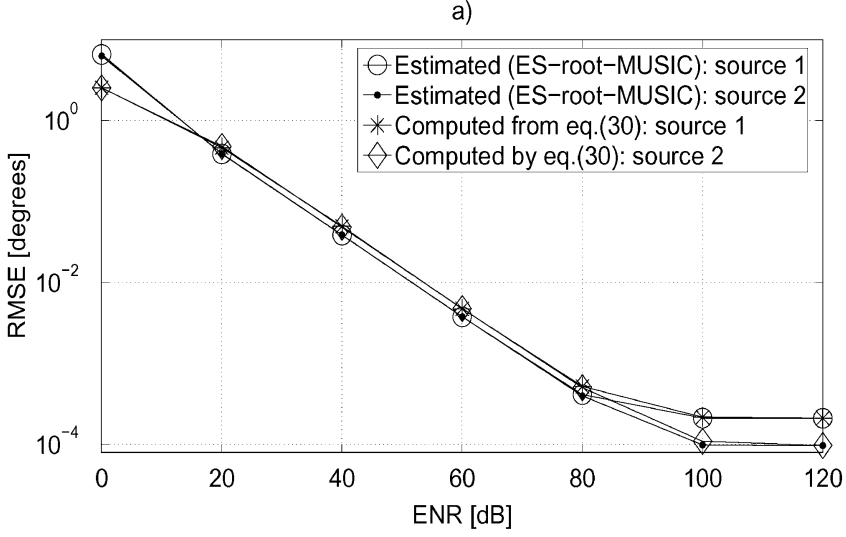

b)

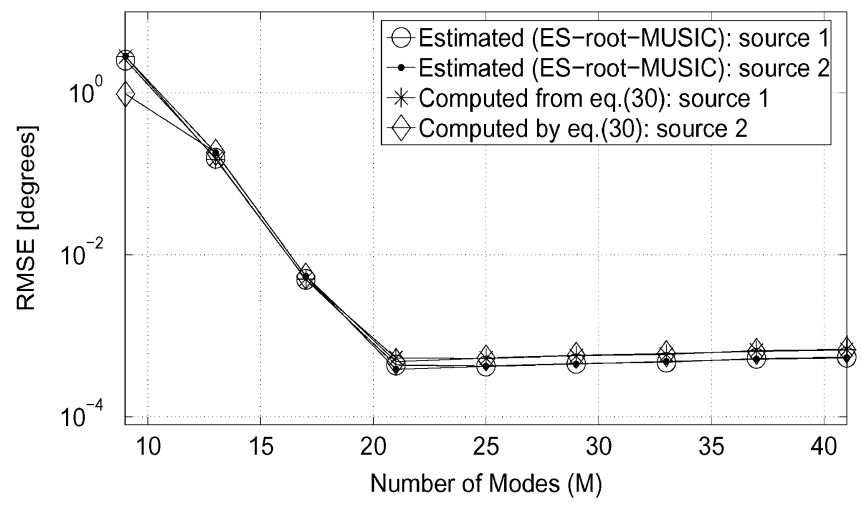

Fig. 4. Comparison between the estimated and computed RMSEs by using ES-root-MUSIC and (30) with $\left(\phi_{1}, \phi_{2}\right)=\left(25^{\circ}, 45^{\circ}\right)$. In (a), the error decreases as the calibration noise decreases (and ENR increases). The error floor is due to the truncation of $\widetilde{\mathbf{G}}_{e}$ to $M=21$ modes. In (b), using an ENR $=80 \mathrm{~dB}$, the minimum of the curve defines the optimum number of modes $M_{\eta}=21$. In both cases, the estimated RMSEs are well approximated by (30).

It converges to a constant value. It can be seen as the rate of change of $\mathbf{E}_{n}^{H} \mathbf{G}_{e} \mathbf{d}_{i}$ about the true DoA.

c) From (a) and (b), we also note that for large enough ENR and $M, \mathbf{E}_{n}^{H} \overline{\mathbf{G}} \mathbf{d}_{i} \approx 0$ and the following inequality holds:

$$
\left|\mathbf{d}_{i}^{\prime \prime H} \overline{\mathbf{G}} \mathbf{E}_{n} \mathbf{E}_{n}^{H} \overline{\mathbf{G}} \mathbf{d}_{i}\right|<\mathbf{d}_{i}^{\prime H} \overline{\mathbf{G}} \mathbf{E}_{n} \mathbf{E}_{n}^{H} \overline{\mathbf{G}} \mathbf{d}_{i}^{\prime} .
$$

The second term in the denominator of (26) dominates.

As a result, (26) can be reduced into the following expression:

$$
\left(\hat{\phi}_{i}-\phi_{i}\right) \approx-\frac{\Re\left\{\mathbf{d}_{i}^{\prime H} \overline{\mathbf{G}}^{H} \mathbf{E}_{n} \mathbf{E}_{n}^{H} \overline{\mathbf{G}} \mathbf{d}_{i}\right\}}{\mathbf{d}_{i}^{\prime H} \overline{\mathbf{G}}^{H} \mathbf{E}_{n} \mathbf{E}_{n}^{H} \overline{\mathbf{G}} \mathbf{d}_{i}^{\prime}} .
$$

In order to verify the approximation (30), we have considered $L=1000$ independent realizations of the calibration noise $\overline{\mathbf{W}}$ for each SNR. We have compared the estimated (see Section VI) and computed RMSEs of the DoAs using ES-root-MUSIC and $\left(\sum_{l=1}^{L}\left(\hat{\phi}_{i, l}-\phi_{i}\right)^{2} / L\right)^{(1 / 2)}$, respectively. In Fig. 4, we show that (30) well describes the error either as a function of ENR or $M$. In the simulations, we have used two uncorrelated sources at $\left(\phi_{1}, \phi_{2}\right)=\left(25^{\circ}, 45^{\circ}\right)$, a UCA with $N=8, r=0.5 \lambda$, and observation noise-free data.

Observe, from an application point of view, (30) is not very practical. It requires the knowledge of the span of the noise subspace $\mathbf{E}_{n}$. The key idea in the next section is to drop this de- 
pendency by deriving an approximate closed-form expression of the MSE due to calibration noise.

\section{B. Multiple Calibration Measurements}

Let us consider measuring an infinite number of times a given antenna array with the same calibration setup (SNR, antennas positions, etc.). This scenario can be modeled similarly to Section VI, but now $\widetilde{\mathbf{G}}_{e}, \widetilde{\mathbf{G}}$ and $\widetilde{\mathbf{b}}\left(\phi, \mathbf{W}, M_{\eta}\right)$ are all random quantities depending on the calibration noise $\mathbf{W}$.

Here, we derive an approximate expression of the MSE due to calibration noise, which depends only on quantities that are known or computable in real-world application, such as the perturbed EADF $\overline{\mathbf{G}}$, the calibration noise power $\sigma_{W}^{2}$, and the number of selected modes $M$. This is of interest in practical applications because it allows us to compute the performance limit achievable by using a perturbed EADF (computed from real-world calibration data) as a model for the antenna array.

As discussed in Section VI, the modes of the noise-free and noisy EADFs with magnitude larger than the measurement noise level are dominated by their expected value; see also Figs. 2 and 3. Consequently, selecting $M$ modes and assuming $\|\mathbf{G}\|_{F} \gg\|\mathbf{W}\|_{F}$, the approximation $\left\|\mathbf{E}_{n}^{H} \widetilde{\mathbf{G}} \mathbf{d}_{i}^{\prime}\right\|^{2} \approx\left\|\mathbf{E}_{n}^{H} \mathbf{G} \mathbf{d}_{i}^{\prime}\right\|^{2}$ holds [29]. In other words, the rates of change of these functions are similar; see also Section VII-A, consideration b.

As a result, we express the approximate MSE of (30) as

$$
\mathrm{E}_{W}\left\{\left(\hat{\phi}_{i}-\phi_{i}\right)^{2}\right\} \approx \frac{\mathrm{E}_{W}\left\{\left(\Re\left\{\mathbf{d}_{i}^{\prime H} \mathbf{G}^{H} \mathbf{E}_{n} \mathbf{E}_{n}^{H} \widetilde{\mathbf{G}} \mathbf{d}_{i}\right\}\right)^{2}\right\}}{\left(\mathbf{d}_{i}^{\prime H} \mathbf{G}^{H} \mathbf{E}_{n} \mathbf{E}_{n}^{H} \mathbf{G d}_{i}^{\prime}\right)^{2}} .
$$

Here, $\mathrm{E}_{W}\{\cdot\}$ stands for expected value with respect to $\mathbf{W}$. Following the derivation in Appendix I, an approximate close-form expression of the MSE can be written as

$$
\mathrm{E}_{W}\left\{\left(\hat{\phi}_{i}-\phi_{i}\right)^{2}\right\} \approx \frac{\sigma_{W}^{2}}{\mathbf{d}_{i}^{\prime H} \overline{\mathbf{G}}^{H} \overline{\mathbf{E}}_{n} \overline{\mathbf{E}}_{n}^{H} \overline{\mathbf{G}} \mathbf{d}_{i}^{\prime}}
$$

where $\overline{\mathbf{E}}_{n}$ is the perturbed noise subspace defined in (38).

In Fig. 5, we compare the estimated RMSEs and computed from ES-root-MUSIC and (32), respectively. Recalling the discussion in Section VI-B, we can observe that expression (32) approximates the RMSE well, when the term Wd dominates the error in the perturbed $\widetilde{\mathbf{b}}\left(\phi, \mathbf{W}, M_{\eta}\right)$.

In the simulations, we have used two uncorrelated sources at $\left(\phi_{1}, \phi_{2}\right)=\left(25^{\circ}, 45^{\circ}\right)$, a UCA with $N=8, r=0.5 \lambda$, and observation noise-free data. Similarly to Section VII-A, we have used 1000 independent realizations of $\mathbf{W}$ for each ENR to estimate the RMSE of ES-root-MUSIC. For the computation of (32), the error floor estimate shown in the same figure, we have used a single realization $\overline{\mathbf{G}}$ for each ENR value.

\section{Simulation RESUlts}

In a real-world scenario, the array calibration measurements are always corrupted by noise. Consequently, the ideal (nonper- a)

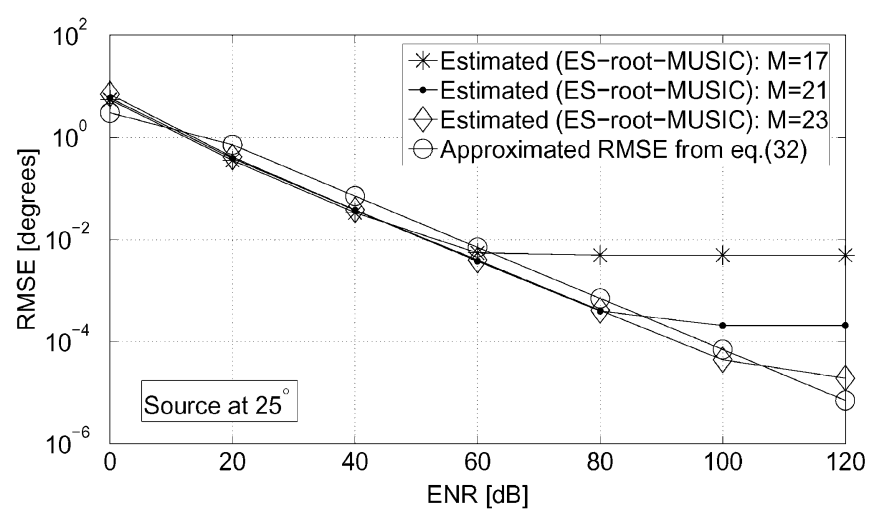

b)

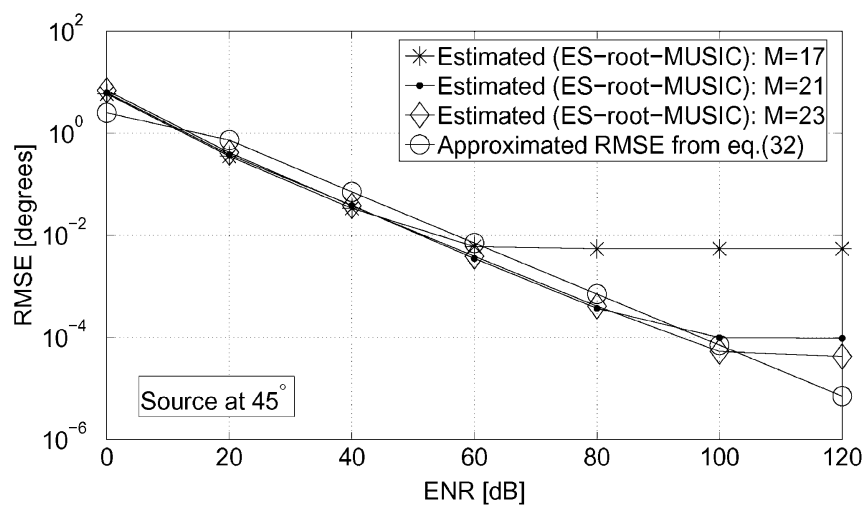

Fig. 5. Comparison between the estimated and computed RMSEs by using ES-root-MUSIC and (32), respectively. The proposed closed-form expression well estimates the saturation floors due to the truncation error. In (a) and (b), the behavior of sources at $\left(\phi_{1}, \phi_{2}\right)=\left(25^{\circ}, 45^{\circ}\right)$ is shown.

turbed) EADF matrix $\mathbf{G}_{e}$ is always an unknown quantity. Here, we have simulated an ideal and perturbed version of the EADF according to the model defined in (17).

In order to verify the accuracy of the proposed error analysis, we compare the expressions in (30) and (32) with the statistical performance of ES-root-MUSIC. Note that both modeling errors and finite sample effects are here considered.

In particular, we have used an UCA with $N=8$ elements and radius $r=0.5 \lambda$ measured by uniformly taking $Q=360$ calibration points in $\phi=[-\pi, \pi)$ with an ENR $=50 \mathrm{~dB}$. The resulting noisy and ideal EADFs are similar to the ones depicted in Fig. 1. We have used 1000 independent realizations of $\mathbf{W}$ and $\mathbf{N}$. The perturbed EADF $\widetilde{\mathbf{G}}$ is then constructed by truncating at $M_{\eta}=17$ modes. The simulation has been carried out considering two uncorrelated sources at $\left(\phi_{1}, \phi_{2}\right)=\left(25^{\circ}, 45^{\circ}\right)$, and $K=256$ observations. Note that (32) has been evaluated using a single realization $\overline{\mathbf{G}}$ for each $\mathrm{SNR}$ value.

From Fig. 6, we observe that, even if the MST uses a perturbed and truncated version of the EADF, for SNRs $<32 \mathrm{~dB}$, the finite sample effects dominate over the antenna modeling error. As a result, the statistical performance of the ES-rootMUSIC algorithm is close to the Cramér-Rao bound (CRB) [9].

On the other hand, when SNRs $>32 \mathrm{~dB}$ the modeling error dominates over the observation noise and finite sample effects 

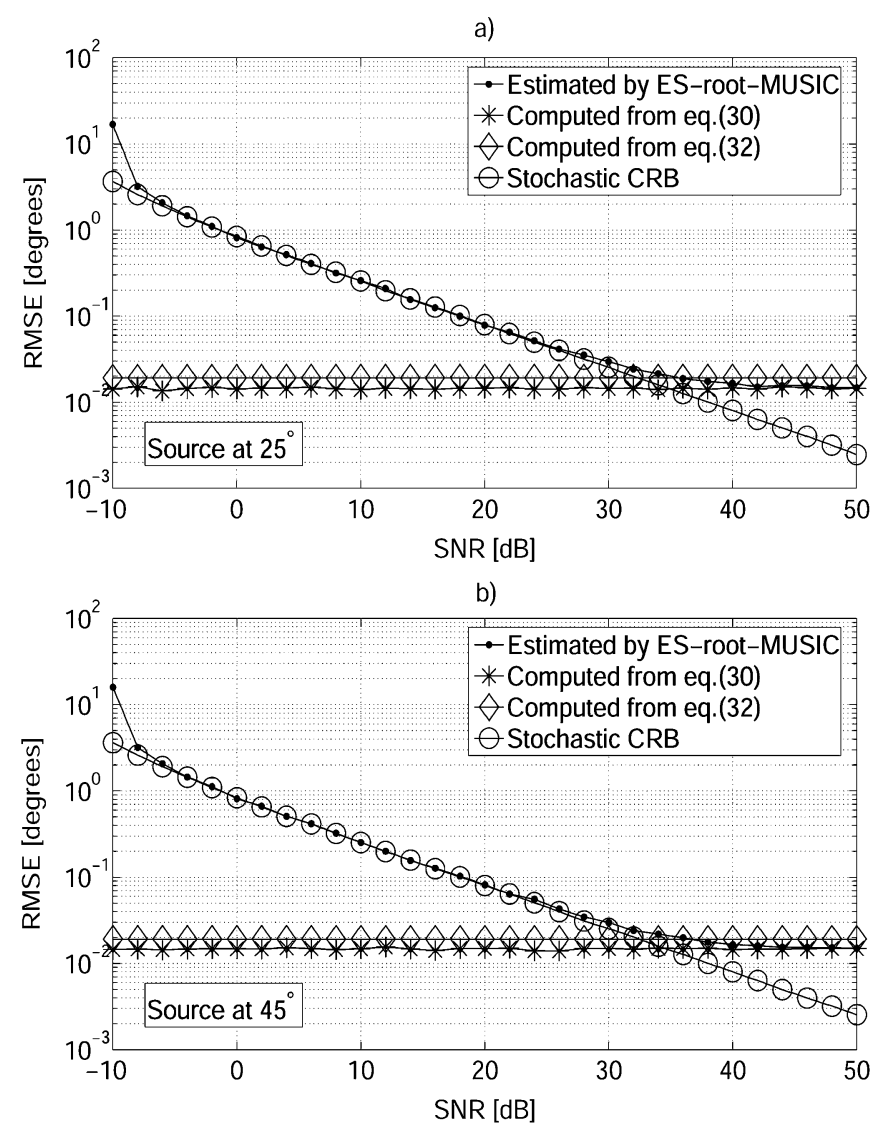

Fig. 6. Comparison between the RMSEs of the DoA estimates using the ES-root-MUSIC and the proposed error analysis. The proposed expressions in (30) and (32) well estimate the error floor due to modeling error. In (a) and (b), simulation results for sources at $\left(\phi_{1}, \phi_{2}\right)=\left(25^{\circ}, 45^{\circ}\right)$.

\section{TABLE II}

NUMERICAL EvaluATION OF THE ERROR FloORS IN Fig. 6 COMPUTED BY USING ES-ROOT-MUSIC, (30) AND (32). THE FLOOR IS WELL APPROXIMATED BY BOTH OF THE PROPOSED EXPRESSIONS

\begin{tabular}{|c|c|c|c|}
\hline & Estimated Error & Error from eq. (30) & Error from eq. (32) \\
\hline Source 1 & 0.0147 & 0.0144 & 0.0192 \\
\hline Source 2 & 0.0148 & 0.0150 & 0.0193 \\
\hline
\end{tabular}

errors. The statistical performance of the DoA estimates saturates. We observe that both expressions (30) and (32) predict the error floor due to modeling errors well.

Note, since the proposed expressions in (30) and (32) are derived under observation noise-free assumptions, they do not depend on the SNR of the sources. From Fig. 6, an interesting observation can be made: The intersection of the CRB and either one of the curves in (30) or (32) defines the operational area of any subspace-based algorithms using MST with the considered array configuration.

A numerical evaluation of the error floor in the DoA estimates using ES-root-MUSIC algorithm and the expressions in (30) and (32) is given in Table II. The proposed analysis accurately describe and predict the error due to calibration noise and truncation of the EADF.

\section{CONCLUSION}

In this paper, we considered the manifold separation technique (MST). We described a practical approach for performing MST through the computation of the EADF. This is based on the IDFT of array calibration data. The proposed preprocessing techniques allow fast direction-of-arrival (DoA) algorithms designed for linear arrays to be used on real-world arrays with arbitrary configuration.

In the first part of this work, we have studied the effect of noisy calibration measurement on both the EADF and the MSE of the DoA estimates. A criterion for selecting the optimum number of modes $M_{\eta}$ has been proposed, and a link between the number of selected modes and the statistics of the calibration noise has been established.

We have analyzed the impact of using a perturbed EADF with MST. The perturbed EADF introduces a modeling error that limits the statistical performance of subspace-based DoA estimation algorithms. Two closed-form expressions describing the error floor have been presented. The latter expression is of particular interest in practical applications because it depends only on quantities which are known or computable in real-world scenarios.

Based on the proposed analysis, it is possible to predict the performance achievable by subspace-based algorithms using MST with noisy calibration data. This may also be done offline. Simulation results showing the accuracy of the proposed analysis have been presented.

\section{APPENDIX I}

DERIVATION OF (32)

By expanding the numerator of (31) and considering (27) and (28), we can write

$$
\begin{aligned}
& \mathrm{E}_{W}\left\{\left(\Re\left\{\mathbf{d}_{i}^{\prime H} \mathbf{G}^{H} \mathbf{E}_{n} \mathbf{E}_{n}^{H} \widetilde{\mathbf{G}} \mathbf{d}_{i}\right\}\right)^{2}\right\} \\
& =\mathrm{E}_{W}\left\{\left(\Re\left\{\mathbf{d}_{i}^{\prime H} \mathbf{G}^{H} \mathbf{E}_{n} \mathbf{E}_{n}^{H}(\mathbf{G}+\mathbf{W}) \mathbf{d}_{i}\right\}\right)^{2}\right\} \\
& \approx \mathrm{E}_{W}\left\{\left(\Re\left\{\mathbf{d}_{i}^{\prime}{ }^{H} \mathbf{G}^{H} \mathbf{E}_{n} \mathbf{E}_{n}^{H} \mathbf{W} \mathbf{d}_{i}\right\}\right)^{2}\right\} .
\end{aligned}
$$

In (33), we have considered that since $\mathbf{E}_{n}^{H} \mathbf{G d}\left(\phi_{i}\right) \approx 0$, the following inequality holds:

$$
\left|\mathbf{d}_{i}^{\prime H} \mathbf{G}^{H} \mathbf{E}_{n} \mathbf{E}_{n}^{H} \mathbf{G d}_{i}\right|<\left|\mathbf{d}_{i}^{\prime}{ }^{H} \mathbf{G}^{H} \mathbf{E}_{n} \mathbf{E}_{n}^{H} \mathbf{W} \mathbf{d}_{i}\right| .
$$

By defining the noise subspace projector $\mathbf{\Pi}_{n}=\mathbf{E}_{n} \mathbf{E}_{n}^{H}$, can then be rewritten as

$$
\begin{aligned}
\mathrm{E}_{W}\left\{\left(\Re\left\{\mathbf{d}_{i}^{\prime H} \mathbf{G}^{H} \boldsymbol{\Pi}_{n} \mathbf{W} \mathbf{d}_{i}\right\}\right)^{2}\right\} \\
=\mathbf{d}_{i}^{\prime H} \mathbf{G}^{H} \boldsymbol{\Pi}_{n} \mathrm{E}_{W}\left\{\mathbf{W} \mathbf{d}_{i} \mathbf{d}_{i}^{H} \mathbf{W}^{H}\right\} \mathbf{\Pi}_{n} \mathbf{G} \mathbf{d}_{i}^{\prime},
\end{aligned}
$$

where

$$
\begin{aligned}
\mathrm{E}_{W} & \left\{\mathbf{W d}_{i} \mathbf{d}_{i}^{H} \mathbf{W}^{H}\right\} \\
= & \mathrm{E}_{W}\left\{\left(\mathbf{d}_{i}^{T} \otimes \mathbf{I}\right) \operatorname{vec}\{\mathbf{W}\} \operatorname{vec}\{\mathbf{W}\}^{H}\left(\mathbf{d}_{i}^{*} \otimes \mathbf{I}\right)\right\} \\
= & \left(\mathbf{d}_{i}^{T} \otimes \mathbf{I}\right) \mathrm{E}_{W}\left\{\operatorname{vec}\{\mathbf{W}\} \operatorname{vec}\{\mathbf{W}\}^{H}\right\}\left(\mathbf{d}_{i}^{*} \otimes \mathbf{I}\right) \\
= & \sigma_{W}^{2}\left(\mathbf{d}_{i}^{T} \otimes \mathbf{I}\right)\left(\mathbf{d}_{i}^{*} \otimes \mathbf{I}\right)=\sigma_{W}^{2} \mathbf{I} .
\end{aligned}
$$


Here, $\operatorname{vec}\{\cdot\}$ denotes the vector operator, and $(\cdot)^{T}$ and $(\cdot)^{*}$ stand for transpose and conjugate, respectively.

Combining (31), (35), and (36), and since $\mathbf{E}_{n}^{H} \mathbf{E}_{n}=\mathbf{I}$, we can express the approximated MSE by

$$
\mathrm{E}_{W}\left\{\left(\hat{\phi}_{i}-\phi_{i}\right)^{2}\right\} \approx \frac{\sigma_{W}^{2}}{\mathbf{d}_{i}^{\prime H} \mathbf{G}^{H} \mathbf{E}_{n} \mathbf{E}_{n}^{H} \mathbf{G d}_{i}^{\prime}} .
$$

Let us denote the perturbed noise subspace by $\overline{\mathbf{E}}_{n} \in$ $\mathbb{C}^{N \times(N-P)}$. This quantity can be computed by eigenvalue decomposition of the $N \times N$ perturbed covariance matrix

$$
\overline{\mathbf{R}}_{x}=\overline{\mathbf{G D D}}^{H} \overline{\mathbf{G}}^{H}=\left[\begin{array}{ll}
\overline{\mathbf{E}}_{s} & \overline{\mathbf{E}}_{n}
\end{array}\right]\left[\begin{array}{cc}
\bar{\lambda}_{s} & \mathbf{0} \\
\mathbf{0} & \mathbf{0}
\end{array}\right]\left[\begin{array}{c}
\overline{\mathbf{E}}_{s}^{H} \\
\overline{\mathbf{E}}_{n}^{H}
\end{array}\right] .
$$

From (27), we note that asymptotically (ENR and $M \rightarrow \infty$ ) $\overline{\mathbf{E}}_{n}$ and $\mathbf{E}_{n}$ spans the same space. Similarly to (31), assuming $\|\mathbf{G}\|_{F} \gg\|\mathbf{W}\|_{F}$, the approximation $\left\|\mathbf{d}_{i}^{\prime H} \mathbf{G}^{H} \mathbf{E}_{n}\right\|^{2} \approx$ $\left\|\mathbf{d}_{i}^{\prime H} \overline{\mathbf{G}}^{H} \overline{\mathbf{E}}_{n}\right\|^{2}$ also holds [29]. By using this approximation in (37), we get the same as in (32).

\section{REFERENCES}

[1] A. J. Barabell, "Improving the resolution performance of eigenstructure-based direction-finding algorithm," in Proc. IEEE int. Conf. Acoustics, Speech, Signal Processing (ICASSP), Apr. 1983, vol. 8, pp. 336-339.

[2] B. Friedlander, "The root-MUSIC algorithm for direction finding with interpolated arrays," Signal Process., vol. 30, pp. 15-29, 1993.

[3] P. Hyberg, M. Jansson, and B. Ottersten, "Array interpolation and bias reduction," IEEE Trans. Signal Process.., vol. 52, no. 10, pp. 2711-2720, Oct. 2004.

[4] P. Hyberg, M. Jansson, and B. Ottersten, "Array interpolation and DOA MSE reduction," IEEE Trans. Signal Process.., vol. 53, no. 12, pp. 4464-4471, Dec. 2005.

[5] B. K. Lau, "Applications of antenna arrays in third-generation mobile communications systems" Ph.D. dissertation, Curtin University of Technology, Perth, Australia, 2002 [Online]. Available: http://adt. curtin.edu.au/theses/available/adt-WCU20030701.132324/

[6] C. P. Mathews and M. D. Zoltowski, "Eigenstructure techniques for 2-D angle estimation with uniform circular arrays," IEEE Trans. Signal Process., vol. 42, no. 9, pp. 2395-2407, Sep. 1994.

[7] F. Belloni and V. Koivunen, "Beamspace transform for UCA: Error analysis and bias reduction," IEEE Trans. Signal Process., vol. 54, no. 8, pp. 3078-3089, Aug. 2006.

[8] F. Belloni, A. Richter, and V. Koivunen, "Reducing excess variance in beamspace methods for uniform circular array," presented at the IEEE Workshop. Statist. Signal Process. (SSP), Bordeaux, France, Jul. 17-20, 2005.

[9] F. Belloni, A. Richter, and V. Koivunen, "Performance of root-MUSIC algorithm using real-world arrays," presented at the 14th Eur. Signal Processing Conf. (EUSIPCO), Florence, Italy, Sep. 4-8, 2006.

[10] F. Belloni, A. Richter, and V. Koivunen, "Extension of root-MUSIC to non-ULA array configurations," presented at the IEEE Int. Conf. Acoustics, Speech, Signal Processing (ICASSP), France, May 14-19, 2006.

[11] A. Richter, F. Belloni, and V. Koivunen, "DoA and polarization estimation using arbitrary polarimetric array configurations," presented at the IEEE Workshop Sensor Array and Multichannel Processing (SAM), Waltham, MA, Jul. 12-14, 2006.

[12] M. A. Doron, E. Doron, and A. J. Weiss, "Coherent wideband processing for arbitrary array geometry," IEEE Trans. Signal Process., vol. 41, no. 1, pp. 414-417, 1993.

[13] M. A. Doron and E. Doron,"Wavefield modeling and array processing, Part I-Spatial sampling," IEEE Trans. Signal Process.., vol. 42, no. 10, pp. 2549-2559, Oct. 1994.
[14] M. A. Doron and E. Doron, "Wavefield modeling and array processing, Part II-Algorithms," IEEE Trans. Signal Process., vol. 42, no. 10, pp. 2560-2570, Oct. 1994.

[15] M. A. Doron and E. Doron, "Wavefield modeling and array processing, Part III-Resolution capacity," IEEE Trans. Signal Process., vol. 42, no. 10, pp. 2571-2580, Oct. 1994.

[16] S. Nemirovsky and M. A. Doron, "Sensitivity of MUSIC and RootMUSIC to gain calibration errors of 2-D arbitrary array configuration," in Proc. 3rd IEEE Sensor Array Multichannel Signal Process. Workshop (SAM), Barcelona, Spain, Jul. 2004, pp. 594-598.

[17] R. S. Thomä, M. Landmann, G. Sommerkorn, and A. Richter, "Multidimensional high-resolution channel sounding in mobile radio," in Proc. IEEE Instrumentation Measurement Tech. Conf. (IMTC), May 2004, vol. 1, pp. 257-262.

[18] A. Richter, "Estimation of radio channel parameters: Models and algorithms" Ph.D. dissertation, Technische Universität Ilmenau, Ilmenau, Germany, 2005 [Online]. Available: www.db-thueringen.de

[19] M. Pesavento, C. F. Mecklenbräuker, and J. F. Böhme, "Multidimensional rank reduction estimator for parametric MIMO channel models," in EURASIP J. Appl. Signal Process., 2004, vol. 9, pp. 1354-1363.

[20] E. W. Weisstein, "Jacobi-anger expansion," Wolfram MathWorld [Online]. Available: mathworld.wolfram.com/Jacobi-AngerExpansion.html

[21] M. Landmann and G. Del Galdo, "Efficient antenna description for MIMO channel modelling and estimation," in Proc. Eur. Conf. Wireless Technology, 2004, pp. 217-220.

[22] G. Del Galdo, J. Lotze, M. Landmann, and M. Haardt, "Modelling and manipulation of polarimatric antenna beam patterns via spherical harmonics," presented at the 14th Eur. Signal Processing Conf. (EUSIPCO), Florence, Italy, Sep. 4-8, 2006.

[23] M. D. Zoltowski, M. Haardt, and C. P. Mathews, "Closed-form 2-D angle estimation with rectangular arrays in element space or beamspace via unitary ESPRIT," IEEE Trans. Signal Process., vol. 44, no. 2, pp. 316-328, Feb. 1996.

[24] A. L. Swindlehurst and T. Kailath, "A performance analysis of subspace-based methods in the presence of model errors. I. The MUSIC algorithm," IEEE Trans. Signal Process., vol. 40, no. 7, pp. 1758-1774, Jul. 1992

[25] B. D. Rao and K. V. S. Hari, "Performance analysis of root-music," IEEE Trans. Signal Process., vol. 37, no. 12, pp. 1939-1949, Dec. 1989.

[26] M. Kaveh and A. Barabell, "The statistical performance of the MUSIC and the minimum-norm algorithms in resolving plane waves in noise," IEEE Trans. Signal Process., vol. 34, no. 2, pp. 331-341, Apr. 1986.

[27] P. Stoica and A. Nehorai, "Music, maximum likelihood, and Cramer-Rao bound," IEEE Trans. Signal Process., vol. 37, no. 5, pp. 720-741, May 1989.

[28] A. Ferréol, P. Larzabal, and M. Viberg, "On the asymptotic performance analysis of subspace DOA estimation in the presence of modeling errors: Case of Music," IEEE Trans. Signal Process., vol. 54, no. 3, pp. 907-920, Mar. 2006

[29] G. W. Stewart and J. G. Sun, Matrix Perturbation Theory. New York: Academic, 1990

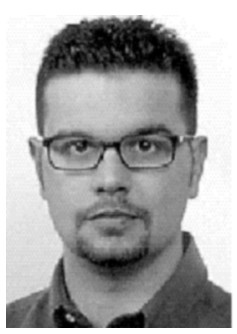

Fabio Belloni (S'03) was born in Castellanza (VA), Italy, in 1978. He received the M.Sc.(Tech.) degree in telecommunication engineering from Politecnico di Milano, Milan, Italy, in 2003. He is currently working towards the Ph.D. degree at the Helsinki University of Technology (TKK), Finland.

Since September 2002, he has been with the Signal Processing Laboratory, Department of Electrical and Communication Engineering, TKK, Finland. Since October 2005, he has been a member of GETA (Graduate school in Electronics, Telecommunications and Automation). His research interests are in the field of multiantenna communications, channel-parameters estimation, and array signal processing. 


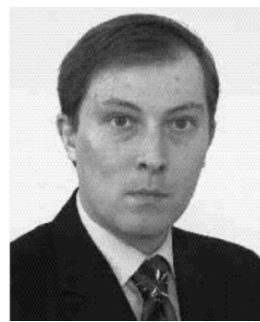

Andreas Richter (M'04) was born in Germany in 1969. He received the Dipl.-Ing. (M.Sc.) degree in electrical engineering and the Dr.-Ing. (Ph.D.) degree (summa cum laude) from the Technische Universität Ilmenau, Ilmenau, Germany, in 1995 and 2005, respectively.

From 1995 to 2005 , he worked as a Research Assistant at the Electronic Measurement Laboratory of Technische Universität Ilmenau. From July to October 2001, he was a Guest Researcher at the Wireless Laboratories, NTT DoCoMo, Yokosuka, Japan. Since 2004, he has been working as a Senior Researcher in the Statistical Signal Processing Laboratory at Helsinki University of Technology, Finland. His research interests are in the fields of digital communication, sensor-array, and statistical signal processing.

Dr. Richter was coauthor or author of five papers receiving a Best Paper Award (EPMCC'01, ISAP'04, PIMRC'05, EUSIPCO'06, and EuCAP'06). In 2005, he received the Siemens Communications Academic Award. He and his former colleagues at Technische Universität Ilmenau received the Thuringien Research Award for Applied Research in 2007 for their work on MIMO channel sounding.

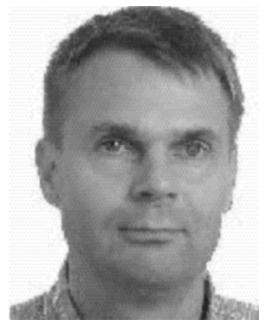

Visa Koivunen (S'87-M'87-SM'98) received the D.Sc.(Tech.) degree with honors from the Department of Electrical Engineering, University of Oulu, Oulu, Finland.

From 1992 to 1995 , he was a visiting researcher at the University of Pennsylvania, Philadelphia. From 1997 to 1999, he was an Associate Professor at the Signal Processing Laboratory, Tampere University of Technology, Tampere, Finland. Since 1999, he has been a Professor of Signal Processing at the Department of Electrical and Communications Engineering, Helsinki University of Technology (TKK), Finland. He is one of the Principal Investigators in SMARAD (Smart and Novel Radios) Center of Excellence in Radio and Communications Engineering nominated by the Academy of Finland. Since 2003, he has been Adjunct Professor at the University of Pennsylvania, Philadelphia. During his sabbatical term 2006-2007, he is a Nokia Visiting Fellow at Nokia Research Center, Helsinki, and visiting fellow at Princeton University, Princeton, NJ. His research interest include statistical, communications, and sensor array signal processing. He has published more than 200 peer-reviewed papers in international scientific conferences and journals.

Dr. Koivunen received the Primus Doctor (best graduate) Award among the doctoral graduates from 1989 to 1994 . He coauthored papers that received the Best Paper Awards in IEEE PIMRC 2005, EUSIPCO 2006, and EuCAP 2006 conferences. He served as an Associate Editor for the IEEE SIGNAL PROCESSING LETTERS. He is a member of the editorial board for the Signal Processing journal and the Journal of Wireless Communication and Networking. $\mathrm{He}$ is also a member of the IEEE Signal Processing for Communication Technical Committee (SPCOM-TC). He is the General Chair of the IEEE Signal Processing Advances in Wireless Communication (SPAWC) 2007 conference in Helsinki, Finland, in June 2007. 